\title{
Universiteit
}

Leiden

The Netherlands

\section{Chronic frequent headache in the general population: comorbidity and quality of life}

Wiendels, N.J.; Haestregt, A. van; Knuistingh Neven, A.; Spinhoven, P.; Zitman, F.G.;

Assendelft, W.J.J.; Ferrari, M.D.

\section{Citation}

Wiendels, N. J., Haestregt, A. van, Knuistingh Neven, A., Spinhoven, P., Zitman, F. G., Assendelft, W. J. J., \& Ferrari, M. D. (2006). Chronic frequent headache in the general population: comorbidity and quality of life. Cephalalgia, 26, 1443-1450.

doi:10.1111/j.1468-2982.2006.01211.x

Version: $\quad$ Not Applicable (or Unknown)

License: $\quad$ Leiden University Non-exclusive license

Downloaded from: https://hdl.handle.net/1887/13184

Note: To cite this publication please use the final published version (if applicable). 


\title{
Chronic frequent headache in the general population: comorbidity and quality of life
}

\author{
NJ Wiendels ${ }^{1,2}$, A van Haestregt ${ }^{1}$, A Knuistingh Neven ${ }^{2}$, P Spinhoven ${ }^{3}$, FG Zitman ${ }^{4}$, WJJ Assendelft ${ }^{2} \&$ \\ MD Ferrari ${ }^{1}$ \\ Departments of ${ }^{1}$ Neurology, ${ }^{2}$ General Practice and Nursing Home Medicine, ${ }^{3}$ Psychology and ${ }^{4}$ Psychiatry, Leiden University Medical Centre, \\ Leiden, the Netherlands
}

\section{Cephalalgia}

Wiendels NJ, van Haestregt A, Knuistingh Neven A, Spinhoven P, Zitman FG, Assendelft WJJ \& Ferrari MD. Chronic frequent headache in the general population: comorbidity and quality of life. Cephalalgia 2006; 26:1443-1450. London. ISSN 0333-1024

We studied the nature and extent of comorbidity of chronic frequent headache $(\mathrm{CFH})$ in the general population and the influence of $\mathrm{CFH}$ and comorbidity on quality of life. Subjects with CFH (headache on $>14$ days/month) were identified in a general health survey. We sent a second questionnaire including questions on comorbidity and quality of life to subjects with $\mathrm{CFH}$ and subjects with infrequent headache (IH) (1-4 days/month). We recoded comorbidity by using the Cumulative Illness Rating Scale (CIRS) and measured quality of life with the RAND-36, a Dutch version of Short Form-36. CFH subjects $(n=176)$ had higher comorbidity scores than the IH subjects $(n=141)$. Mean CIRS scores were 2.94 for $\mathrm{CFH}$ and 1.55 for $\mathrm{IH}$ [mean difference $1.40,95 \%$ confidence interval (CI) 0.91, 1.89]. The mean number of categories selected was 1.92 in $\mathrm{CFH}$ and $1.10 \mathrm{in} \mathrm{IH}$ (mean difference $0.82,95 \%$ CI $0.54,1.11$ ). Fifty percent of CFH subjects had a comorbidity severity level of at least 2 , indicating disorders requiring daily medication, compared with $28 \%$ of IH subjects (mean difference $22 \%, 95 \%$ CI 12 , 33). CFH subjects had more musculoskeletal, gastrointestinal, psychiatric and endocrine/breast pathology than IH subjects. Quality of life in CFH subjects was lower than that of IH subjects in all domains of the RAND-36. Both headache frequency and CIRS score had a negative influence on all domains. We conclude that patients with $\mathrm{CFH}$ have more comorbid disorders than patients with infrequent headaches. Many CFH patients have a comorbid chronic condition requiring daily medication. Both high headache frequency and comorbidity contribute to the low quality of life in these patients. $\square$ Chronic daily headache, comorbidity, headache, medication overuse, quality of life

N. J. Wiendels MD, Department of Neurology, K5Q-106, Leiden University Medical Centre, PO Box 9600, 2300 RC Leiden, the Netherlands. Tel. + 3171526 1730, fax +3171524 8253, e-mail n.j.wiendels@lumc.nl Received 18 November 2005, accepted 15 June 2006

\section{Introduction}

Epidemiological studies on comorbidity of headache disorders have focused primarily on psychiatric disorders. Migraine has been repeatedly found to be associated with major depression and anxiety disorders (1-4). Breslau et al. found a bi-directional relationship between migraine and major depression; a history of major depression is a risk factor for migraine and migraine increases the risk for major depression (3). They suggested that shared underlying factors explain the co-occurrence of the two disorders, rather than major depression being a psychological response to recurrent severe headaches.

Chronic frequent headache (CFH), also known as chronic daily headache, is defined as headache on 
$>14$ days per month for at least 3 months. Around $4 \%$ of the general population suffer from CFH $(5,6)$. In headache clinics, the majority of patients with $\mathrm{CFH}$ have a comorbid psychiatric disorder (7-9). The most commonly reported disorders are major depression and generalized anxiety disorder, followed by panic disorder and phobias. High headache frequency and chronic substance use are associated with higher scores on anxiety and depression scales $(10,11)$. In a general population sample, aged $>64$ years, a high score on a depression scale was associated with $\mathrm{CFH}$ and subjects with $\mathrm{CFH}$ were at increased risk of major depression at follow-up (12).

Few studies have explored comorbidity of headache with somatic disorders. Associations of migraine with epilepsy, stroke, asthma and chronic musculoskeletal pain have been reported (13-15). $\mathrm{CFH}$ has been associated with allergies, asthma, hypothyroidism, hypertension, sleep disorders and fibromyalgia $(16,17)$. These studies were conducted in headache clinics, which might have led to an overestimation of associations, because referred patients may represent a selected, difficult to treat population. To avoid this bias, population-based studies are preferred.

The overall comorbidity of $\mathrm{CFH}$ in the general population has not been studied systematically. Co-occurrence of diseases can complicate diagnosis, due to symptomatic overlap of the two conditions, and can have important implications for treatment. Moreover, comorbidity can have a negative influence on quality of life. Quality of life is reduced in subjects with $\mathrm{CFH}$ and is greatly influenced by anxiety and depressive disorders (18-20). We studied the nature and extent of comorbidity in $\mathrm{CFH}$ patients in the general population and examined the influence of $\mathrm{CFH}$ and comorbidity on quality of life.

\section{Methods}

We conducted a general health survey amongst all persons, aged 25-55 years, registered at 16 general practices in the province of South-Holland in the Netherlands in 2003. This sample represents the general population because in the Netherlands all individuals are registered at a general practice. The study design and methodology have been described in detail previously (21). In short, 76\% completed the general health survey. We identified subjects with $\mathrm{CFH}$, defined as headache on $>14$ days per month during the past 3 months, and sent them a second, more detailed questionnaire containing questions on comorbidity and quality of life. Forty percent completed the questionnaire. Non-responders showed no relevant demographic differences from the responders. A random sample of subjects with infrequent headache $(\mathrm{IH})$, defined as headache on 1-4 days per month, served as the control group. The majority $(62 \%)$ of subjects with $\mathrm{CFH}$ overused acute headache medication. This study was conducted before the publication of the revised International Headache Society criteria for medication overuse headache. We defined overuse as: the use of analgesics on $\geq 3$ days/week, the use of triptans on $\geq 2$ days/week, the use of ergots on $\geq 1$ day/week or the use of narcotics on $\geq 10$ days / month. Overuse consisted mainly of analgesics, only three $(2 \%)$ overused triptans, one ergotamine and seven $(4 \%)$ narcotics.

Comorbidity was assessed by the following open questions: (i) 'Do you have a disorder for which you have to consult your physician regularly?'; (ii) 'Do you have other disorders for which regular consultation is not necessary at the moment?'; (iii) 'Have you been admitted to hospital in the past? If yes, please specify'; (iv) 'Which medication (including painkillers) do you use?'. We recoded answers by using the Cumulative Illness Rating Scale (CIRS) (22). The CIRS is a reliable and validated comorbidity questionnaire and shows close resemblance to common clinical practice: it is structured according to 14 body systems and uses a clear severity ranking that is clinically sound (23-27). The worst problem in a specific organ system is rated on a scale from 0 to 4 ( 0 , none; 1 , current mild problem or significant problem in the past; 2, moderate disability/requires daily medication; 3, severe/ constant disability, uncontrollable chronic problem; and 4, extremely severe/immediate treatment required/end organ failure/severe impairment in function). Five summary scores can be calculated [total CIRS score, total number of categories endorsed, severity index (total score/total number of categories) and number of level 3 and 4 severity] (22). The total CIRS score was our main outcome measure. Headache, our index disease, was not rated as a comorbid disorder. Whenever the manual of the CIRS was not clear about how to rate a certain symptom or disease, we rated the symptom by consensus and used a data file to record our decisions. We modified the psychiatric illness rating as follows: current usage of daily antidepressants or anxiolytics without sleeping problems was rated as severity 2, and psychiatric illness with daily use of two medications as severity 3. Frequent sleeping problems were listed under neurological comorbidity, with occasional use of hypnotics as severity 1 
and daily use of hypnotics as 2 . Use of benzodiazepines without a specified indication was rated according to their registered indication (e.g. diazepam as anxiolytic).

Quality of life was measured by the RAND-36, a Dutch version of the RAND-36-Item Short Form Health Survey, a commonly used generic quality of life questionnaire (28). The RAND-36 has been shown to have excellent reliability and validity when employed with diverse patient populations in the Netherlands (29). It consists of eight domains of well-being and functioning, including Physical Functioning (PF), Social Functioning (SF), Physical Role Functioning (PRF), Emotional Role Functioning (ERF), Mental Health (MH), Vitality (V), Bodily Pain (BP) and General Health (GH), and an additional item, Health Transition (HT). The scales range from 0 to 100, with higher scores indicating better quality of life.

Data analysis was performed using SPSS 11.0 (SPSS Inc., Chicago, IL, USA). Differences are presented with $95 \%$ confidence intervals (95\% CI). Differences in comorbidity categories and quality of life domains between $\mathrm{CFH}$ subjects and $\mathrm{IH}$ subjects were tested for significance. Due to multiple comparisons we applied a Bonferroni adjustment yielding an $\alpha$ level of 0.003 for the CIRS categories and 0.006 for the RAND-36 domains (30). The relationship between RAND-36 scores and CIRS scores was investigated using Spearman's rank order correlation. Values between 0.10 and 0.29 were considered to indicate a weak correlation, between 0.30 and 0.49 a medium strong correlation and between 0.50 and 1.0 a strong correlation. Hierarchical multiple regression was used to examine the relationship between each RAND-36 domain and headache frequency (case status) while controlling for educational level and CIRS score.

\section{Results}

Table 1 shows the demographic characteristics of both headache groups. The CFH group had more subjects with a lower educational level. The presence of comorbidity is summarized in Table 2. One CFH subject did not complete the comorbidity section of the questionnaire and was excluded from analysis. In both headache groups, the majority currently had or had had in the past at least one comorbid problem. Of $149 \mathrm{CFH}$ subjects with any comorbidity, $60 \%$ had a severity level of at least 2 , indicating disorders requiring daily medication (e.g. hypertension).

In both groups the most prevalent comorbid disorders were in the gastrointestinal and musculosketal/skin categories. In the $\mathrm{CFH}$ group 21 of 24 subjects $(88 \%)$ with upper gastrointestinal problems reported heartburn, 19 used antacids or acid suppressants, six of them in combination with non-steroidal anti-inflammatory drugs (NSAIDs). In the IH group four of seven subjects (57\%) used acid suppressants. Lower gastrointestinal problems were mainly appendectomies in the past-16 (9\%) CFH, five $(4 \%) \mathrm{IH}$; or other operations-seven $(4 \%) \mathrm{CFH}$, seven $(5 \%) \mathrm{IH}$. In the musculoskeletal/skin category, joint operations in the past-19 (11\%) CFH, $14(10 \%) \mathrm{IH}$, and arthritis; $10(6 \%) \mathrm{CFH}$, five $(4 \%)$ $\mathrm{IH}$ were the most commonly reported disorders, followed by back pain-nine (5\%) CFH, six (4\%) IH; and neck pain/whiplash-seven (4\%) CFH, $0 \mathrm{IH}$. Six $\mathrm{CFH}$ subjects (3\%) reported fibromyalgia, none in the IH group. Dermatological disorders were reported only once in the $\mathrm{CFH}$ group and four times in the IH group.

CFH subjects had more endocrine/breast, psychiatric and neurological pathology than the IH subjects. The endocrine/breast group consisted of a sum of several disorders: thyroid pathology, diabetes and breast cancer. In the psychiatry group, the following comorbid problems contributed most to the ratings: depressive mood or current use of antidepressants-11 (6\%) CFH, two (1\%) IH; anxiety disorder or current use of anxiolytics-13 $(7 \%) \mathrm{CFH}$, three $(2 \%) \mathrm{IH}$; current use of both antidepressants and anxiolytics-five (3\%) $\mathrm{CFH}$, one $(0 \%) \mathrm{IH}$. The differences in neurological ratings were mainly due to sleeping problems or current use of hypnotics-22 (13\%) CFH, nine $(6 \%) \mathrm{IH}$.

Table 1 Demographic characteristics of $\mathrm{CFH}$ and $\mathrm{IH}$ groups

\begin{tabular}{|c|c|c|c|}
\hline & $\begin{array}{l}\text { CFH } \\
N=177\end{array}$ & $\begin{array}{l}\mathrm{IH} \\
N=141\end{array}$ & $\begin{array}{l}\text { Difference, } \\
\%(95 \% \text { CI })\end{array}$ \\
\hline Mean age, years (SD) & $43(8.4)$ & $42(8.0)$ & $0.5(-1.5,2.4)$ \\
\hline Female, $n(\%)$ & $125(72)$ & $97(70)$ & $2 \%(-8,12)$ \\
\hline Low educational level, $n(\%)$ & $62(35)$ & $16(11)$ & $24 \%(15,33)$ \\
\hline
\end{tabular}

$\mathrm{CFH}$, Chronic frequent headache (>14 days/month); IH, infrequent headache (1-4 days/month). 
Table 2 Presence of comorbidity in the CFH group vs. IH group

\begin{tabular}{lccc}
\hline & CFH, $n=176$ & IH, $n=141$ & Difference, \% (95\% CI) \\
\hline Presence of any comorbidity & $149(85)$ & $91(65)$ & $20(11,29)^{*}$ \\
Comorbidity with severity level $\geq 2$ & $88(50)$ & $39(28)$ & $22(12-33)^{*}$ \\
Comorbidity with severity level $\geq 3$ & $29(17)$ & $13(9)$ & $7(0,15)$ \\
Comorbidity categories endorsed & & & \\
Heart & $6(3)$ & $3(2)$ & $1(-2,5)$ \\
Vascular & $24(14)$ & $17(12)$ & $2(-6,9)$ \\
Haematopoietic & $4(2)$ & $2(1)$ & $1(-2,4)$ \\
Respiratory & $19(11)$ & $7(5)$ & $6(0,12)$ \\
Eyes, ears, nose and throat & $33(19)$ & $19(14)$ & $5(-3,14)$ \\
Gastrointestinal & $54(31)$ & $23(16)$ & $14(5,24)^{*}$ \\
$\quad 24(14)$ & $7(5)$ & $9(2,15)^{*}$ \\
$\quad$ Upper gastrointestinal & $37(21)$ & $16(11)$ & $10(1,18)^{*}$ \\
Liver & $7(4)$ & $2(1)$ & $3(-1,6)$ \\
Renal & $4(2)$ & $4(3)$ & $-1(-4,3)$ \\
Genitourinary & $24(14)$ & $16(11)$ & $2(-5,10)$ \\
Musculoskeletal/skin & $60(34)$ & $32(23)$ & $11(1,21)^{*}$ \\
Neurological & $41(23)$ & $18(13)$ & $11(2,19)^{*}$ \\
Endocrine/breast & $21(12)$ & $3(2)$ & $10(4,16)^{* \dagger}$ \\
Psychiatric & $33(19)$ & $8(6)$ & $13(6,20)^{* \dagger}$ \\
\hline
\end{tabular}

Values are $n(\%)$.

${ }^{*} 95 \% \mathrm{CI}$ excludes the neutral value of no difference $(0 \%)$.

$\dagger$ Bonferroni: $P<0.003$.

$\mathrm{CFH}$, Chronic frequent headache; $\mathrm{IH}$, infrequent headache.

Table 3 CIRS scores

\begin{tabular}{lccc}
\hline & CFH, $n=176$ & IH, $n=141$ & Difference (95\% CI) \\
\hline Total CIRS score & $2.94(2.52)$ & $1.54(1.75)$ & $1.40(0.91,1.89)$ \\
Number of categories endorsed & $1.92(1.42)$ & $1.10(1.10)$ & $0.82(0.54,1.11)$ \\
Severity index & $1.47(0.48)$ & $1.36(0.51)$ & $0.11(-0.02,0.23)$ \\
Comorbidity with severity level 3, $n(\%)$ & $27(15)$ & $13(9)$ & $6 \%(-1,14)$ \\
Comorbidity with severity level $4, n(\%)$ & $2(1)$ & $0(0)$ & $1 \%(-1,3)$
\end{tabular}

Values are means (SD), unless stated otherwise.

CIRS, Cumulative Illness Rating Scale; CFH, chronic frequent headache; IH, infrequent headache.

Other reported disorders were epilepsy-six (3\%) $\mathrm{CFH}$; hernia-five (3\%) $\mathrm{CFH}$, three $(2 \%) \mathrm{IH}$; and miscellaneous disorders-eight (5\%) CFH, six (4\%) IH. In both psychiatric and endocrine/breast categories the difference between CFH subjects and IH subjects was significant at the adjusted $\alpha$ level of 0.003 , but not in the gastrointestinal, musculoskeletal and neurological categories.

CFH subjects had higher total CIRS scores than IH subjects (Table 3 ). Median number of categories endorsed was two in the CFH group vs. one in the IH group. CFH was associated with a CIRS comorbidity level of at least 2 with a crude odds ratio of 2.6 (95\% CI 1.6, 4.2), and adjusted for educational level $2.2(95 \%$ CI $1.3,3.5)$. In the CFH group over- users had higher total CIRS scores than nonoverusers (Table 4), but severity was not significantly higher.

Quality of life of CFH subjects was lower in all domains of the RAND-36 compared with the IH subjects (Fig. 1). Scores for Health Transition were 45.2 in the $\mathrm{CFH}$ group and 54.6 in the $\mathrm{IH}$ group, mean difference -9.4 (95\% CI $-14.3,-4.5)$. Small differences in quality of life between subjects who overused acute headache medication and those who did not were not statistically significant (Fig. 2). All domains of the RAND-36 were negatively correlated with CIRS score (Table 5). Hierarchical multiple regression showed that after controlling for educational level and CIRS score, 
Table 4 CIRS scores in overusers compared with non overusers

\begin{tabular}{lccc}
\hline & Overuse, $n=109$ & No overuse, $n=67$ & Difference, (95\% CI) \\
\hline Total CIRS score & $3.34(2.62)$ & $2.28(2.21)$ & $1.06(0.30,1.81)$ \\
Number of categories endorsed & $2.11(1.43)$ & $1.60(1.35)$ & $0.51(0.08,0.94)$ \\
Severity index & $1.51(0.50)$ & $1.38(0.44)$ & $0.13(-0.03,0.29)$ \\
Comorbidity severity level $\geq 2, n(\%)$ & $61(56)$ & $27(40)$ & $16 \%(0,31)$ \\
Comorbidity severity level 3, $n(\%)$ & $21(24)$ & $6(9)$ & $15 \%(-1,14)$ \\
Comorbidity severity level $4, n(\%)$ & $2(2)$ & $0(0)$ & $2 \%(-1,5)$ \\
\hline
\end{tabular}

Values are means (SD), unless stated otherwise.

CIRS, Cumulative Illness Rating Scale.

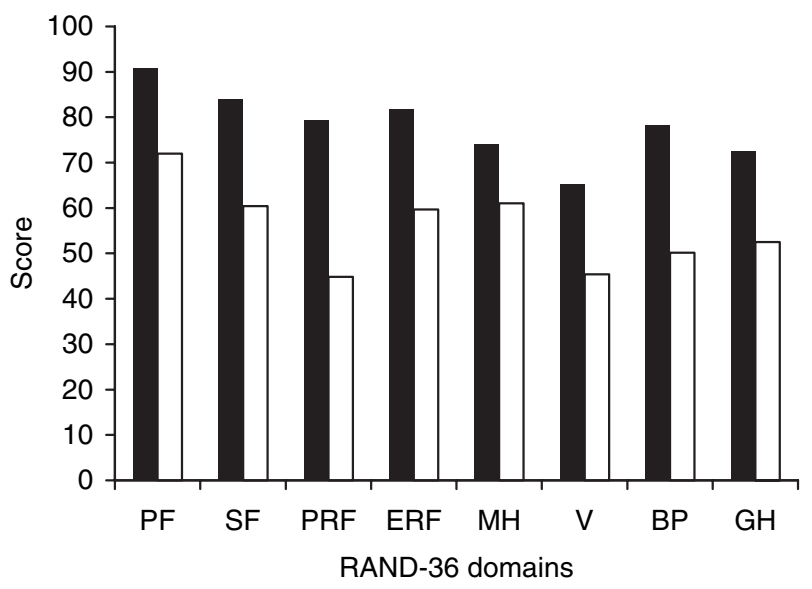

Figure 1 Mean RAND-36 scores (28) in the chronic frequent headache $(\mathrm{CFH}, \square)(n=173)$ and infrequent headache $(\mathrm{IH}, \mathbf{\square})(n=141)$ groups. $\mathrm{CFH}$ differs significantly from $\mathrm{IH}$ in all domains $(P<0.001)$. PF, Physical functioning; SF, social functioning; PRF, physical role functioning; ERF, emotional role functioning; $\mathrm{MH}$, mental health; V, vitality; $\mathrm{BP}$, bodily pain; $\mathrm{GH}$, general health.

$\mathrm{CFH}$ case status remained an independent predictor of all RAND-36 domains. Case status explained 5\% (Emotional Role Functioning) to 25\% (Bodily Pain) of the variance.

\section{Discussion}

To the best of our knowledge, the overall comorbidity of $\mathrm{CFH}$ has not previously been studied systematically in a population-based sample. Subjects with CFH reported more somatic and psychiatric comorbidity than subjects with infrequent headaches. Overusers reported more comorbidity than non-overusers. Fifty percent of the CFH subjects had a comorbid problem causing at least moderate disability or requiring daily medication (severity level $\geq 2$ ).

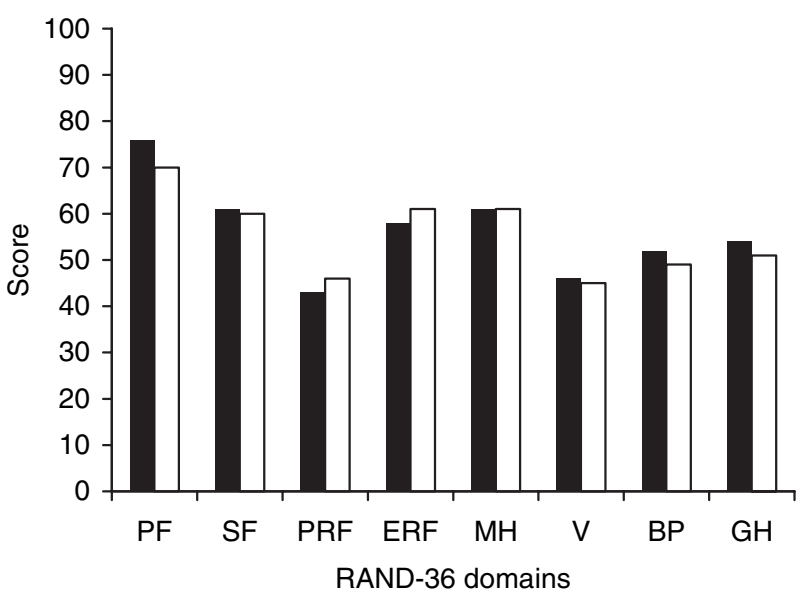

Figure 2 Mean RAND-36 scores (28) in the chronic frequent headache $(\mathrm{CFH})$ group with $(\square, n=107)$ and without $(\boldsymbol{\square}, n=66)$ overuse of acute headache medication. Differences are not statistically significant. PF, Physical functioning; SF, social functioning; PRF, physical role functioning; ERF, emotional role functioning; $\mathrm{MH}$, mental health; V, vitality; $\mathrm{BP}$, bodily pain; $\mathrm{GH}$, general health.

The CIRS is meant to give a global estimate of medical burden taking into account both the presence and the severity of disorders. Its accuracy depends on the ability of subjects to report diseases. Self-reports have proven to be reasonably accurate for estimations of prevalent health conditions (24, 31). The number of comorbid problems in our study was lower than reported in other studies using the CIRS $(24,25)$. This is probably due to the lower mean age of our participants and the populationbased setting. We think our results are a reliable estimate of the overall prevalence of comorbidity in headache patients in the general population. Twenty-eight percent of subjects in the IH group had a comorbid disorder of at least severity level 2. This is in accordance with another study conducted in the Netherlands (32), where the prevalence of 
Table 5 Relationship between quality of life and comorbidity

\begin{tabular}{ll}
\hline $\begin{array}{l}\text { Quality of life: RAND-36 } \\
\text { domains }\end{array}$ & $\begin{array}{l}\text { Comorbidity: correlations } \\
\text { with CIRS score }\end{array}$ \\
\hline Physical Functioning & $-0.47^{*}$ \\
Social Functioning & $-0.29^{*}$ \\
Physical Role Functioning & $-0.28^{*}$ \\
Emotional Role Functioning & $-0.20^{*}$ \\
Mental Health & $-0.24^{*}$ \\
Vitality & $-0.31^{*}$ \\
Bodily Pain & $-0.34^{*}$ \\
General Health & $-0.50^{*}$
\end{tabular}

Values are Spearman's $\rho$.

$* P<0.001$.

CIRS, Cumulative Illness Rating Scale (22); RAND-36, quality of life questionnaire (28).

two or more co-occurring chronic or recurrent diseases within one person in general practice was $29.7 \%$.

Musculoskeletal problems were frequently reported. Although not significant after Bonferroni correction, chronic pain conditions tended to be present more frequently in $\mathrm{CFH}$ than in $\mathrm{IH}$ subjects. Headache has been associated with musculoskeletal pain in the general population before; migraine was associated with chronic back pain, and low back pain in adolescents with headache $(15,33)$. In a headache clinic, Peres et al. diagnosed fibromyalgia in $36 \%$ of chronic migraine patients (16). Although we did not find such a high prevalence, six CFH subjects reported fibromyalgia vs. none in the $\mathrm{IH}$ group. Central sensitization, a pathological change in central pain processing, could play a role in the co-occurrence of these chronic pain conditions (34).

Many $\mathrm{CFH}$ subjects reported gastrointestinal problems. Heartburn, a symptom of gastrooesophageal reflux disease, was the most frequently reported upper gastrointestinal disorder. It is a common health problem and has considerable impact on quality of life (35). Heartburn in the CFH group could not be attributed entirely to NSAID gastropathy since only $25 \%$ of $\mathrm{CFH}$ subjects with heartburn used NSAIDs. The higher gastrointestinal comorbidity in CFH subjects compared with $\mathrm{IH}$ subjects might reflect a higher anxiety and perceived stress level in CFH subjects (36).

The prevalence of psychiatric disorders in $\mathrm{CFH}$ in the general population has never been studied. In the elderly in Taiwan, CFH was associated with high scores on a depression scale (12). Studies in headache clinics have shown that $\mathrm{CFH}$ was associated with depression and anxiety disorders (7-9). It was not surprising, therefore, to find more psychiatric pathology in the CFH group than in the $\mathrm{IH}$ group. However, the percentage of subjects with psychiatric comorbidity in the $\mathrm{CFH}$ group was lower than expected. We previously found that the majority of our $\mathrm{CFH}$ subjects screen positive for psychopathology on the General Health Questionnaire (21). The prevalence of self-reported psychiatric comorbidity may have been underestimated due to hesitation to report psychiatric problems or simply because they may not yet have been diagnosed. The true prevalence of psychiatric disorders can be assessed only by an interview based on Diagnostic and Statistical Manual of Mental Disorders (DSM) criteria.

Endocrine/breast disorders were present more frequently in the $\mathrm{CFH}$ group than in the IH group. The endocrine/breast category consisted of various disorders, the numbers being too small for meaningful subanalyses.

The RAND-36 scores in the IH group were similar to normal values. Quality of life is clearly impaired in the CFH group. Physical Role Functioning and Vitality scored the lowest. The difference between $\mathrm{CFH}$ and IH was most marked for Physical Role Functioning and Bodily Pain. The Physical Role Functioning domain measures whether one cannot fulfil one's role/work because of a physical problem. Considering the high prevalence of $\mathrm{CFH}$, the low score in this particular domain implies that CFH not only affects a subject's personal life but also has a major impact on society. Our results are comparable to those of other studies. In Spain, two studies found similar decreases of quality of life measured with the SF-36 in subjects with $\mathrm{CFH}$ in the general population. Both Colas et al. and Guitera et al. found Physical Role Functioning, Bodily Pain and Vitality to be the most affected domains $(19,20)$. Guitera et al. also found that subjects with analgesic overuse scored lower than the non-overusers in all domains, especially Physical Functioning and Bodily Pain. In our study the small differences between overusers and non-overusers were not statistically significant, indicating that overuse causes only minor to no extra impairment. Both headache frequency and comorbidity had a negative influence on quality of life. Logically, headache frequency showed a strong influence on Bodily Pain and CIRS score on General Health.

A limitation of this study is that comorbidity rating is based on open general questions on comorbid disorders. If questions on specific symptoms, such as joint or back pain, had been included, 
we assume that certain body systems (e.g. musculoskeletal) might have scored higher. Our data also lacked information on various objective parameters such as body mass index and levels of serum cholesterol and haemoglobin, data that would surely have increased endocrine, vascular and haematopoietic comorbidity scores. However, we did not aim to find specific disease prevalence rates or associations, but rather to form an impression of the extent of comorbidity experienced by and thus most likely proving burdensome to the headache patient. Causal relationships cannot be proved by this cross-sectional study.

In conclusion, $\mathrm{CFH}$ patients in the general population have more somatic and psychiatric comorbidity than patients with infrequent headaches. Many $\mathrm{CFH}$ patients have a comorbid chronic condition requiring daily medication. Both high headache frequency and comorbidity contribute to the low quality of life in these patients. The relatively high prevalence of $\mathrm{CFH}$, the high comorbidity rate and the low quality of life indicate that $\mathrm{CFH}$ is a major health problem.

\section{Competing interests}

None declared.

\section{Acknowledgements}

This study was supported by a grant of the Netherlands Organization for Scientific Research (NWO 940-31-049) and by the 'Doelmatigheidsfonds van het LUMC' (LUMC fund to increase efficiency in healthcare). We thank the following GPs for their participation: K. F. van de Bent, A. Boels, J. J. F. M. Brinkmann, T. Buiter-van der Kooi, H. A. Dirkse, L. van Duijn, F. M. M. van Exter, F. G. W. M. Haase, H. van Klei, J. Klein Haneveld, W. Libeton, C. H. Luirink, E. H. J. Smids, M. H. C. van der Velden, R. M. Vermeer, R. Voskuil, P. G. Willemse and B. W. de Zeeuw.

\section{References}

1 Merikangas KR, Merikangas JR, Angst J. Headache syndromes and psychiatric disorders: association and familial transmission. J Psychiatr Res 1993; 27:197-210.

2 Swartz KL, Pratt LA, Armenian HK, Lee LC, Eaton WW. Mental disorders and the incidence of migraine headaches in a community sample: results from the Baltimore Epidemiologic Catchment area follow-up study. Arch Gen Psychiatry 2000; 57:945-50.

3 Breslau N, Lipton RB, Stewart WF, Schultz LR, Welch KM. Comorbidity of migraine and depression: investigating potential etiology and prognosis. Neurology 2003; 60:1308-12.

4 McWilliams LA, Goodwin RD, Cox BJ. Depression and anxiety associated with three pain conditions: results from a nationally representative sample. Pain 2004; 111:77-83.

5 Castillo J, Munoz P, Guitera V, Pascual J. Epidemiology of chronic daily headache in the general population. Headache 1999; 39:190-6.

6 Scher AI, Stewart WF, Liberman J, Lipton RB. Prevalence of frequent headache in a population sample. Headache 1998; 38:497-506.

7 Mathew NT, Stubits E, Nigam MP. Transformation of episodic migraine into daily headache: analysis of factors. Headache 1982; 22:66-8.

8 Verri AP, Proietti CA, Galli C, Granella F, Sandrini G, Nappi G. Psychiatric comorbidity in chronic daily headache. Cephalalgia 1998; 18 (Suppl. 21):45-9.

9 Juang KD, Wang SJ, Fuh JL, Lu SR, Su TP. Comorbidity of depressive and anxiety disorders in chronic daily headache and its subtypes. Headache 2000; 40:818-23.

10 Mitsikostas DD, Thomas AM. Comorbidity of headache and depressive disorders. Cephalalgia 1999; 19:211-7.

11 Radat F, Sakh D, Lutz G, el Amrani M, Ferreri M, Bousser MG. Psychiatric comorbidity is related to headache induced by chronic substance use in migraineurs. Headache 1999; 39:477-80.

12 Wang SJ, Fuh JL, Lu SR, Liu CY, Hsu LC, Wang PN, Liu HC. Chronic daily headache in Chinese elderly: prevalence, risk factors, and biannual follow-up. Neurology 2000; 54:314-9.

13 Ottman R, Lipton RB. Comorbidity of migraine and epilepsy. Neurology 1994; 44:2105-10.

14 Welch KM. Relationship of stroke and migraine. Neurology 1994; 44:S33-S36.

15 Terwindt GM, Ferrari MD, Tijhuis M, Groenen SM, Picavet HS, Launer LJ. The impact of migraine on quality of life in the general population: the GEM study. Neurology 2000; 55:624-9.

16 Peres MF, Young WB, Kaup AO, Zukerman E, Silberstein SD. Fibromyalgia is common in patients with transformed migraine. Neurology 2001; 57:1326-8.

17 Bigal ME, Sheftell FD, Rapoport AM, Tepper SJ, Lipton RB. Chronic daily headache: identification of factors associated with induction and transformation. Headache 2002; 42:575-81.

18 Wang SJ, Fuh JL, Lu SR, Juang KD. Quality of life differs among headache diagnoses: analysis of SF-36 survey in 901 headache patients. Pain 2001; 89:285-92.

19 Guitera V, Munoz P, Castillo J, Pascual J. Quality of life in chronic daily headache: a study in a general population. Neurology 2002; 58:1062-5.

20 Colas R, Munoz P, Temprano R, Gomez C, Pascual J. Chronic daily headache with analgesic overuse: epidemiology and impact on quality of life. Neurology 2004; 62:1338-42.

21 Wiendels NJ, Knuistingh Neven A, Rosendaal FR, Spinhoven P, Zitman FG, Assendelft WJJ et al. Chronic frequent headache in the general population: prevalence and associated factors. Cephalalgia 2006; 26:1434-42.

22 Miller MD, Paradis CF, Houck PR, Mazumdar S, Stack JA, Rifai AH et al. Rating chronic medical illness burden in geropsychiatric practice and research: application of the Cumulative Illness Rating Scale. Psychiatry Res 1992; 41:237-48. 
23 de Groot V, Beckerman H, Lankhorst GJ, Bouter LM. How to measure comorbidity: a critical review of available methods. J Clin Epidemiol 2003; 56:221-9.

24 Visser M, Marinus J, van Hilten JJ, Schipper RG, Stiggelbout AM. Assessing comorbidity in patients with Parkinson's disease. Mov Disord 2004; 19:824-8.

25 Extermann M, Overcash J, Lyman GH, Parr J, Balducci L. Comorbidity and functional status are independent in older cancer patients. J Clin Oncol 1998; 16:1582-7.

26 Parmelee PA, Thuras PD, Katz IR, Lawton MP. Validation of the Cumulative Illness Rating Scale in a geriatric residential population. J Am Geriatr Soc 1995; 43:130-7.

27 Waldman E, Potter JF. A prospective evaluation of the cumulative illness rating scale. Aging (Milano) 1992; 4:171-8.

28 Zee KI, Sanderman R. Het meten van de algemene gezondheidstoestand met de RAND-36, handleiding. [Measurement of the general health situation with the RAND-36, manual.] Groningen: Noordelijk centrum voor Gezondheidsvraagstukken 1993.

29 Aaronson NK, Muller M, Cohen PD, Essink-Bot ML, Fekkes M, Sanderman R et al. Translation, validation, and norming of the Dutch language version of the SF-36 Health Survey in community and chronic disease populations. J Clin Epidemiol 1998; 51:1055-68.

30 Bland JM, Altman DG. Multiple significance tests: the Bonferroni method. BMJ 1995; 310:170.
31 Martin LM, Leff M, Calonge N, Garrett C, Nelson DE. Validation of self-reported chronic conditions and health services in a managed care population. Am J Prev Med $2000 ; 18: 215-8$.

32 van den Akker M, Buntinx F, Metsemakers JF, Roos S, Knottnerus JA. Multimorbidity in general practice: prevalence, incidence, and determinants of co-occurring chronic and recurrent diseases. J Clin Epidemiol 1998; 51:367-75.

33 Hestbaek L, Leboeuf-Yde C, Kyvik KO, Vach W, Russell $\mathrm{MB}$, Skadhauge $\mathrm{L}$ et al. Comorbidity with low back pain: a cross-sectional population-based survey of 12- to 22-year-olds. Spine 2004; 29:1483-91.

34 Burstein R, Cutrer MF, Yarnitsky D. The development of cutaneous allodynia during a migraine attack clinical evidence for the sequential recruitment of spinal and supraspinal nociceptive neurons in migraine. Brain 2000; 123:1703-9.

35 Revicki DA, Wood M, Maton PN, Sorensen S. The impact of gastroesophageal reflux disease on health-related quality of life. Am J Med 1998; 104:252-8.

36 Bradley LA, Richter JE, Pulliam TJ, Haile JM, Scarinci IC, Schan CA et al. The relationship between stress and symptoms of gastroesophageal reflux: the influence of psychological factors. Am J Gastroenterol 1993; 88:11-9. 